\title{
Physician Wellbeing: A Critical Deficiency in Resilience Education and Training
}

\author{
Eugene V. Beresin ${ }^{1}$ • Tracey A. Milligan ${ }^{2}$ - Richard Balon ${ }^{3}$ • John H. Coverdale ${ }^{4}$. \\ Alan K. Louie ${ }^{5}$ - Laura Weiss Roberts ${ }^{5}$
}

Received: 1 December 2015 / Accepted: 3 December 2015 /Published online: 21 December 2015

(C) Academic Psychiatry 2015

These are the duties of a physician: First...to heal his mind and to give help to himself before giving it to anyone else.

"From the epitaph of an Athenian doctor, 2 AD [1]."

As physicians, we hold a unique place in society. We are entrusted with the responsibility of healing people and advancing the health of populations. This role is an honor and a privilege, and yet it is one that comes with the cost of jeopardizing our wellbeing. Physicians may be considered an "at risk" population, with higher rates of depression, anxiety, suicide, divorce, stress, and emotional exhaustion than other segments of the overall population. The role played by physicians requires many sacrifices-long hours, isolation from friends and family, psychological stress and responsibility in caring for very sick patients, tolerance of uncertainty, sleep deprivation, and huge economic burdens following many years of costly education, among others.

Threats to the wellbeing of physicians begin early in training. The stresses of medical school, residency, and postgraduate work all involve managing tremendous pressures to

Eugene V. Beresin

eberesin@partners.org

\footnotetext{
Harvard Medical School, Boston, MA, USA

Brigham and Women's Hospital, Boston, MA, USA

Wayne State University, Detroit, MI, USA

4 Baylor College of Medicine, Houston, TX, USA

5 Stanford University, Stanford, CA, USA
}

acquire information, garner skills, develop and integrate a professional identity with the early-career physician's sense of self, and balance work and life. Roughly half of medical students experience burnout over the first 4 years of medical training. Burnout is defined as emotional exhaustion, depersonalization (i.e., treating patients as objects), and feelings of worthlessness. Burnout results in poor self-care and patient care, diminished empathy, medical error, and poor physical health [2]. Among medical students, more than $20 \%$ will suffer from depression within the first 2 years and up to $9 \%$ will have suicidal ideation before graduation [3]. The number of those who are depressed may increase during the first year of residency [4]. Among practicing physicians, the suicide rate is approximately double the rate in the general population [5]. In the USA, approximately one physician dies by suicide every day - thus each year we lose to suicide about the number of graduates from two medical schools.

Many factors contribute to the distress experienced by physicians. Shortages of health care professionals, demanding caseloads, verbal abuse and other belittling or bullying behaviors, tremendous debt following lengthy medical education, increased regulatory pressures, decreased insurance reimbursements for services, and staying current with overwhelming amounts of new knowledge are but a few examples. These challenges are compounded by our profession's hidden curriculum - the reluctance to admit weakness, expose our shame of suffering from the stigma of a psychiatric disorder, or even discuss the pressures we share. It turns out that we are all deeply imperfect and that we will need to overcome much on our paths as physicians.

With greater recognition of the risks associated with becoming and being a physician has also come greater understanding that our profession has, to a great extent, failed to 
provide adequate care for its members. Although the health of physicians and physicians-in-training has been a topic of concern for over three decades, physician wellbeing has only in recent years been identified as a high priority for our profession. Despite sound research on our significant professional risks, self-care has not been routinely and systematically taught in medical schools, nor has it been emphasized for its importance through the developmental lifespan of the physician. A number of controlled trials inform on the efficacy of stress-management programs for medical students [6]; however, there has been a failure to teach and promote resilience.

\section{What Is Resilience?}

Resilience may be defined as the ability of an individual to maintain personal and social stability despite adversity [7]. In the past, resilience was considered a trait - a personal quality or strength that one is born with or develops throughout the course of life. More recent conceptualizations have moved somewhat away from the traditional view of resilience and now consider it a process rather than a trait and, importantly, link it with a skill set that may be learned [5]. Resilience refers to two complementary activities: (1) preventive: warding off hardships by actively resisting adversity and (2) corrective: coping effectively under traumatic situations.

Engagement, attachment, and reflection foster resilience. The more an individual is engaged with others - friends, family, mentors, peers, and other important figures throughout life (for physicians, these include patients) - the more skills are learned, skills that enhance prevention and coping. Such skills include an ability to communicate effectively and to use the help of others toward building means of preventing or dealing effectively with hardship. Engagement with the environment is also critical in resilience, helping individuals be more amenable to a healthy lifestyle. The more an individual is aware of his or her strengths, weaknesses, and vulnerabilities, the better one is positioned to become resilient. Further, awareness of the environment, culture, and other influences in society that may hinder wellbeing is crucial in building resilience.

Zwack and Schweitzer [5] studied physician burnout, looking particularly at the resilience strategies of physicians who demonstrated wellbeing despite the many stresses associated with the profession. These researchers noted that physicians who found satisfaction in active, gratifying engagement with patients, colleagues, family, and friends fared better. Further, those who were more aware of their mental and physical state fostered resilience through activities such as practicing personal reflection - being mindful of their stress level, reactivity, and the impact of past life events on present-day functioning - and recognizing when personal or professional changes were needed. In addition, physicians in their study reported that other helpful aspects of fostering resilience included setting limits on work and work hours, finding time for exercise and leisure activities, and demarcating their personal and professional lives. Epstein and Krasner [8] have argued that an effective means of promoting resilience includes practicing mindfulness to bolster awareness and consequent self-monitoring and self-regulation. Mindfulness may be practiced individually, in activities such as meditation, or in formal, reflective physicians' groups and workshops.

\section{Strengthening Resilience of Physicians}

Resilience may be fostered among physicians, and resilience training ideally should be integrated into undergraduate, graduate, and postgraduate educational requirements. Although we may not be able to radically or rapidly change the social and economic forces impinging on our lives, we certainly could provide improved education and guidance for healthy personal and professional habits and change a longstanding dangerous hidden curriculum. If we are able to promote the value that resilience is a necessity and a core component of professionalism, an attitudinal change among the body of physicians may well influence future medical institutional structures and policies. State-of-the-art and well-evidenced education will impart not only medical knowledge but also a toolbox of coping skills early in medical education. These coping skills should be supported later in residencies as well as in practice. The evidence needs to be marshaled to shift the hidden curriculum so that caring for oneself, maintaining cognitive and emotional acuity, and seeking fulfillment are considered professional obligations and preconditions for optimal patient outcomes.

The good news is that some medical schools, residencies, and practice groups are changing, bringing greater emphasis to physician wellbeing. Various interventions for promoting medical student and physician wellbeing and preventing negative effects such as depression and suicide have been tested. For instance, internists at Mayo Clinic conducted a study examining whether an intervention involving facilitated smallgroup curriculum would improve wellbeing [9]. The intervention involved 19 biweekly, facilitated physician discussion groups incorporating elements of mindfulness, reflection, shared experience, and small-group learning. Protected paid time for participants was provided by the institution. The intervention improved participants' meaning and engagement in work and reduced depersonalization, with sustained results at a 12-month follow-up. Another study [10] demonstrated that a free, easily accessible, brief web-based cognitive-behavioral therapy program helped to reduce the likelihood of suicidal ideation among medical interns.

Such work and interventions foster health among the physician workforce and have been shown to improve patient care practices. Physician satisfaction is associated with the 
perception of being able to provide quality patient care, and there is a strong correlation between physician and patient satisfaction $[11,12]$. Some of the signs of general growth in this area include the following:

- Participating in small, process-oriented, reflective student and resident seminars, and groups that discuss the emotional, physical, and social impacts of the practice of medicine. Ideally these groups are longitudinal and address the stresses of doctoring during the participants' developmental stages.

- Increasing curricular attention to topics such as personal transformation in the course of medical education and training; the impact of an identity shift from "lay person" to "doctor"; challenges in personal and professional lives; effects of the hidden curriculum on personal and professional development; erosion of empathy or personal ethical standards; managing uncertainty and ambiguity; caring for difficult or hateful patients; working in healthy and dysfunctional teams; coping with unsuccessful results; understanding medical error and the need for apology; understanding systems of care; identifying causes of physician "burnout"; reviewing institutional practices that affect burnout; discovering personal sources of renewal; finding meaning in work; and maintaining curiosity despite time pressures, among others.

- Learning the means to become reflective practitioners, including writing and sharing reflection essays, participating in reflective dialogue, and describing personal seminal experiences. Roberts et al. [13] have shown that empathy, compassion, attunement, and communication may be nurtured by reflecting on one's experience with illness, either personally or in a loved one.

- Developing modules on nutrition, exercise, and healthy diet in medical undergraduate and postgraduate education.

- Including the instruction of mindful meditation in the medical education curriculum.

- Promoting opportunities for students, residents, faculty, and clinicians across specialties to strengthen their relationships through discussion groups, conferences, retreats, and social events.

- Providing detailed and ongoing education in the signs and symptoms of burnout and offering resources for receiving professional help.

- Fostering participation in the National Depression Screening Day (held yearly in October, during Mental Illness Awareness Week) and National Anxiety and Depression Awareness Week (annually in the first week of May).

- Including health care humanities in the medical curriculum, because studies in narrative medicine and in the arts have been shown to increase physician mindfulness and personal awareness.

- Developing institutional awareness and resources that support physician wellbeing.
While this is by no means an exhaustive list of the range of resources we need to develop, one thing is clear: If we do not begin to change the culture of medicine and systematically address the wide range of dangers to the health of physicians, the negative trends in our own wellbeing and mortality will continue and potentially worsen. Indeed, not only do we need to prevent harm on ourselves, we need to heal ourselves by promoting resilience and wellness intervention programs for medical trainees and practitioners.

Acknowledgments Much of this material is from the Harvard Medical School curricula "The Developing Physician: Lifelong Integration of Personal and Professional Growth, with Sensitive, Compassionate Care," a required course for first-year students, and "Patient-Doctor III," a required yearlong course for third-year students during their clerkships. Drs. Beresin and Milligan are two directors of these curricula.

\section{Compliance with Ethical Standards}

Disclosure On behalf of all authors, the corresponding author states that there is no conflict of interest.

\section{References}

1. Maas PL, Oliver JH. An ancient poem on the duties of a physician. Bull Hist Med. 1939;7:315-23.

2. Krasner MS, Epstein RM, Beckman H, et al. Association of an educational program in mindful communication with burnout, empathy, and attitudes among primary care physicians. JAMA. 2009;302:1284-93.

3. Goebert D, Thompson D, Takeshita J, et al. Depressive symptoms in medical students and residents: a multischool study. Acad Med. 2009;84:236-41.

4. Sen S, Kranzler HR, Krystal JH, et al. A prospective cohort study investigating factors associated with depression during medical internship. Arch Gen Psychiatry. 2010;67:557-65.

5. Zwack J, Schweitzer J. If every fifth physician is affected by burnout, what about the other four? Resilience strategies of experienced physicians. Acad Med. 2013;88:382-9.

6. Shiralkar MT, Harris TB, Eddins-Folensbee FF, Coverdale J. A systematic review of stress-management programs for medical students. Acad Psychiatry. 2013;37:158-64.

7. Luthar SS, Cicchetti D, Becker B. The construct of resilience: a critical evaluation and guidelines for future work. Child Dev. 2000;71:543-62.

8. Epstein RM, Krasner MS. Physician resilience: what it means, why it matters, and how to promote it. Acad Med. 2013;88: 301-3.

9. West CP, Dyrbye LN, Rabatin JT, et al. Intervention to promote physician well-being, job satisfaction, and professionalism: a randomized clinical trial. JAMA Int Med. 2014;174: 527-33.

10. Guille C, Zhao Z, Krystal J, Nichols B, Brady K, Sen S. Web-based cognitive behavioral therapy intervention for the prevention of suicidal ideation in medical interns: a randomized clinical trial. JAMA Psychiatry. 2015. doi:10.1001/jamapsychiatry.2015.1880. 
11. Goitein L. Physician well-being: addressing downstream effects, but looking upstream. JAMA Int Med. 2014;174:533-4.

12. DeVoe J, Fryer Jr GE, Straub A, McCann J, Fairbrother G. Congruent satisfaction: is there geographic correlation between patient and physician satisfaction? Med Care. 2007;45:88-94.
13. Roberts LW, Warner TD, Moutier C, Geppert CM, Green Hammond KA. Are doctors who have been ill more compassionate? Attitudes of resident physicians regarding personal health issues and the expression of compassion in clinical care. Psychosomatics. 2011;52:367-74. 